\title{
ON THE ARENS PRODUCTS AND REFLEXIVE BANACH ALGEBRAS
}

\author{
PAK-KEN WONG \\ Department of Mathematics \\ Seton Hall University \\ South Orange, New Jersey 07079
}

(Received March 29, 1990)

\begin{abstract}
We give a characterization of reflexive Banach algebras involving the Arens product.
KEY WORDS AND PHRASES. Arens products, Arena regularity, conjugate space, weakly completely continuous (w.c.c.) algebra.
\end{abstract}

1980 AMS SUBJECT CLASSIFICATION CODES. Primary 46H10; Secondary 46H99.

\section{INTRODUCTION.}

Let $\mathrm{A}$ be a semisimple Banach algebra and $A^{* *}$ the second conjugate space of $\mathrm{A}$ with the Arena product $o$. If $\left(A^{* *}, o\right)$ is semisimple and it has a dense socle, then we show that the following statements are equivalent: (1) $\mathrm{A}$ is reflexive. (2) $A^{* *}$ is w.c.c. (3) $\mathrm{A}$ is w.c.c. (4) $\mathrm{A}$ and $A^{* *}$ have the same socle. This is a generalization of a result by Duncan and Hosseinuim $[1, \mathrm{p} .319$, Theorem 6(ii)]. We also show that if $A^{* *}, o$ ) is semisimple and A is l.w.c.c., then A is Arens regular.

2. NOTATION AND PRELIMINARIES. Definitions not explicitly given are taken from Rickart's book [2].

Let A be a Banach algebra. Then $A^{*}$ and $A^{* *}$ will denote the first and second conjugate spaces of $\mathrm{A}$, and $\pi$ the canonical map of $\mathrm{A}$ into $A^{* *}$. The two Arens products on $A^{* *}$ are defined in stages according to the following rules (see [3] and [4]). Let $x, y \in A, f \in A^{*}$, and $F, G \in A^{* *}$.

Define fox by $(f \circ x)(y)=f(x y)$. Then fox $\in A^{* *}$.

Define Gof by $(\mathrm{Gof})(\mathrm{x})=\mathrm{G}(\mathrm{fox})$. Then Gof $\in A^{*}$.

Define FoG by $(\mathrm{FoG})(\mathrm{f})=\mathrm{F}(\mathrm{Gof})$. Then FoG $\in A^{* *}$.

Define xo'f by $\left(x o^{\prime} \mathrm{f}\right)(\mathrm{y})=\mathrm{f}(\mathrm{yx})$. Then xo'f $\in A^{*}$.

Define fo' $\mathrm{F}$ by $\left(\mathrm{fo}^{\prime} \mathrm{F}\right)(\mathrm{x})=\mathrm{F}(\mathrm{xo}, \mathrm{f})$. Then fo' $\mathrm{F} \in A^{*}$.

Define Fo'G by $\left(F_{o}^{\prime} G\right)(f)=G(f o f)$. Then Fo'G $\in A^{* *}$.

$A^{* *}$ is a Banach algebra under the products FoG and Fo'G and $\pi$ is an algebra isomorphism of A into $\left(A^{* *}, o\right)$ and $\left(A^{* *}, o^{\prime}\right)$. In general, o and $o^{\prime}$ are distinct on $A^{* *}$. If they agree on $A^{* *}$, then $\mathrm{A}$ is called Arens regular. 
LEMMA 2.1. Let A be a Banach algebra. Then, for all $x \in A, f \in A^{*}$, and $F, G \in A^{* *}$, we have

(1) $\pi(x) o F=\pi(x) o^{\prime} F$ and $F o \pi(x)=F o^{\prime} \pi(x)$.

(2) If $\left\{F_{t}\right\} \subset A^{* *}$ and $F_{t} \rightarrow F$ weakly in $A^{* *}$, then $F_{t} o G \rightarrow F o G$ and $G o^{\prime} F_{t} \rightarrow G o^{\prime} F$ weakly.

PROOF. See [3, p.842 and p. 843].

Let $\mathrm{A}$ be a Banach algebra. An element a $\in \mathrm{A}$ is called left weakly completely continuous (1.w.c.c.) if the mapping $L_{a}$ defined by $L_{a}(x)=a x(X \in A)$ is weakly completely continuous. We say that $\mathrm{A}$ is 1.w.c.c. if each $a \in A$ is 1.w.c.c. If $\mathrm{A}$ is both 1.w.c.c. and r.w.c.c., then $\mathrm{A}$ is called w.c.c.

In this paper, all algebras and linear spaces under consideration are over the field $\mathrm{C}$ of complex numbers.

\section{THE MAIN RESULT.}

LEMMA 3.1. Let $\mathrm{A}$ be a Banach algebra. Then $\mathrm{A}$ is 1.w.c.c. (resp. r.w.c.c.) if and only $\pi(A)$ is a right (resp. left) ideal of $\left(A^{* *}, o\right)$.

PROOF. This result is well known (see [1, p.318, Lemma 3] or [2, p.443, Lemma]).

In the rest of this section, we shall assume that $\mathrm{A}$ and $\left(A^{* *}, o\right)$ are semisimple Banach algebras.

THEOREM 3.3. Suppose that $\left(A^{* *}, o\right)$ has a dense socle. Then the following statements are equivalent:
(1) $\mathrm{A}$ is reflexive.
(2) $A^{* *}$ is w.c.c.
(3) $\mathrm{A}$ is w.c.c.
(4) $\pi(A)$ and $A^{* *}$ have the same socle.

PROOF.

(1) $\Rightarrow(2)$. Assume that $\mathrm{A}$ is reflexive. Then $A^{(4)}=A^{* *}=A$; in particular, $\pi(A)^{* *}$ is a twosided ideal of $A^{(4)}$. Hence by Lemma $3.1, A^{* *}$ is w.c.c.

$(2) \Rightarrow(3)$. Assume that $A^{* *}$ is w.c.c. Then $\pi\left(A^{* *}\right)$ is a two-sided ideal of $A^{(4)}$. As observed in $[1$, p.319, Theorem $6(\mathrm{ii})], \pi(A)$ is a two-sided ideal of $A^{* *}$. Hence $\mathrm{A}$ is w.c.c.

(3) $\Rightarrow(4)$. Assume that $\mathrm{A}$ is w.c.c. Then $\pi(A)$ is a two-sided ideal of $A^{* *}$. Let $\mathrm{E}$ be a minimal idempotent of $A^{* *}$. Since $E o A^{* *} o E=E o \pi(A) o E=C E$, it follows that $E \in \pi(A)$. Consequently, $\mathrm{E}$ is a minimal idempotent of $\pi(A)$. If $\mathrm{e}$ is a minimal idempotent of $\mathrm{A}$, then $\pi(e) o A^{* *} \subset \pi(A)$ and so $\pi(e) o A^{* *}=\pi(e A)$. Hence, $\pi(e) o A^{* *} o \pi(e)=\pi(e A e)^{*}=C \pi(e)$ and so $\pi(e)$ is a minimal idempotent of $A^{* *}$. Therefore, $\pi(A)$ and $A^{* *}$ have the same socle.

(4) $\Rightarrow(1)$. Assume that $\pi(A)$ and $A^{* *}$ have the same socle. Since $\pi(S)$ is dense in $A^{* *}$, it follows that $\pi(A)$ is dense in $A^{* *}$ and so $\pi *(A)=A^{* *}$. Therefore $\mathrm{A}$ is reflexive. This completes the proof of the theorem.

REMARK. It is well known that a semisimple annihilator Banach algebra A is w.c.c. (see [5]). Also, A has a dense socle. Therefore, Theorem 3.2 generalizes [1, p.319, Theorem 6(ii)].

THEOREM 3.3. If $A$ is 1.w.c.c., then $A$ is Arens regular.

PROOF. Since $\mathrm{A}$ is 1.w.c.c., by Lemma $3.1, \pi(A)$ is a right ideal of $A^{* *}$. Let $\mathrm{F}$ and $\mathrm{G} \in A^{* *}$ and $x \in A$. Then

$$
\begin{aligned}
\pi(x) o\left(F o G-F o^{\prime} G\right) & =\pi(x) o F o G-\pi(x) o\left(F o^{\prime} G\right) \\
& =\pi(x) o F o G-\pi(x) o^{\prime}\left(f o^{\prime} G\right) \quad \text { By Lemma 2.1(1)) }
\end{aligned}
$$




$$
\begin{aligned}
& =\pi(x) \circ F_{o}\left(i-\left(\pi(x) \circ F^{\prime}\right) o^{\prime}(i\right. \\
& =\pi(x) \circ F^{\prime} \circ(i-(\pi(x) \circ F) \circ G \quad \text { (because } \pi(x) o F \in \pi(A)) \\
& =0
\end{aligned}
$$

Hence $\pi(A) o\left(F o G-F^{\prime} o^{\prime} G\right)=(0)$. Therefore, by Lemma $2.1(2)$, we have $A^{* *} o\left(F o G-F o^{\prime} G\right)=(0)$. Since $\left(A^{* *}, o\right)$ is semisimple, it follows that $F o G-F o^{\prime} G=0$ and so $F o g=F o^{\prime} G$. Therefore, A is Arens regular. This completes the proof.

\section{REFERENCES}

1. DUNCAN, J. and HOSSEINIUM, S.A.R. The Second Dual of a Banach Algebra, Proc. Royal Soc. of Edinburgh. 84A (1979), 309-325.

2. WONG, P.K. Arens Produce and the Algebra of Double Multipliers, Proc. Amer. Math. Soc. $\underline{94}$ (1985), 441-444.

3. ARENS, R.F. The Adjoint of a Bilinear Operation, Proc. Amer. Math. Soc. 2 (1951), 839-848.

4. BONSALL, F.F. and DUNCAN, J. Complete Normed Algebras, Springer, Berlin, 1973.

5. WONG, P.K. On the Arens Product and Certain Banach Algebras, Trans. Amer. Math. Soc. $\underline{180}(1973), 437-438$.

6. RICKART, C.E. General Theory of Banach Algebras, University Series in Higher Math., Van Nostrand, Princeton, N.J., 1960 .

7. WONG, P.K. Arens Product and the Algebra of Double Multipliers II, Proc. Amer. Soc. 100 (1987), 447-453. 


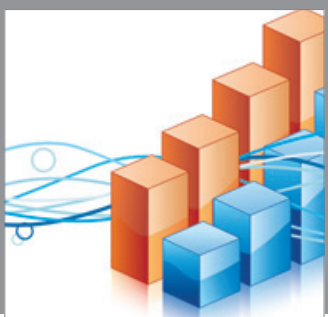

Advances in

Operations Research

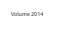

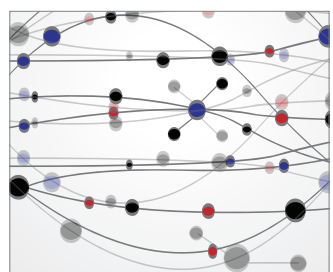

\section{The Scientific} World Journal
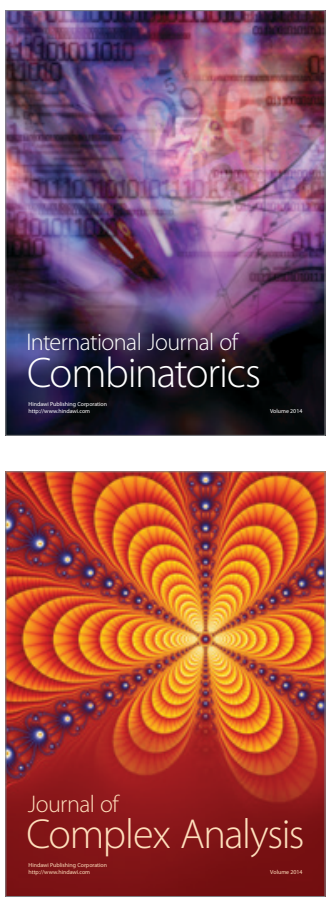

International Journal of

Mathematics and

Mathematical

Sciences
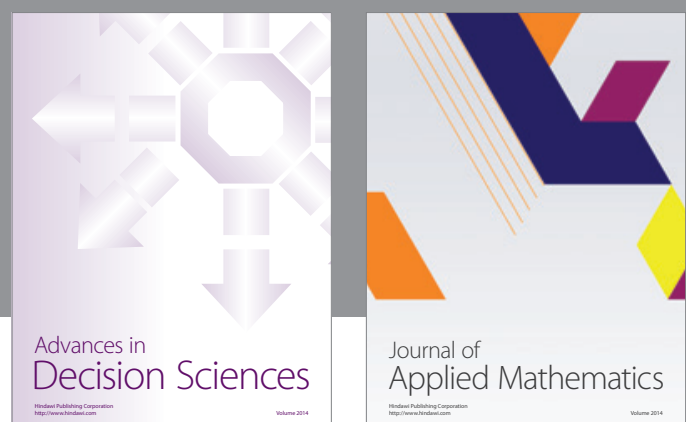

Journal of

Applied Mathematics
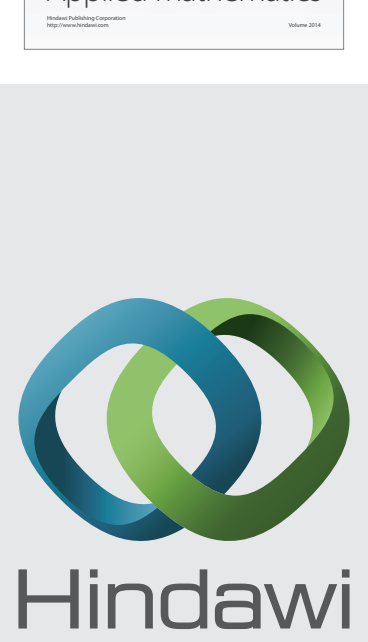

Submit your manuscripts at http://www.hindawi.com
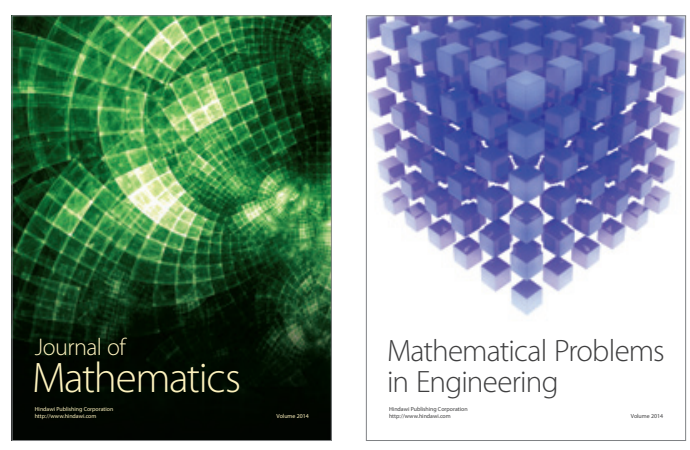

Mathematical Problems in Engineering
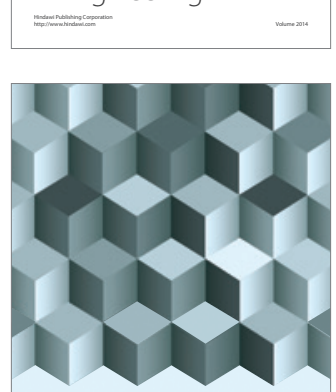

Journal of

Function Spaces
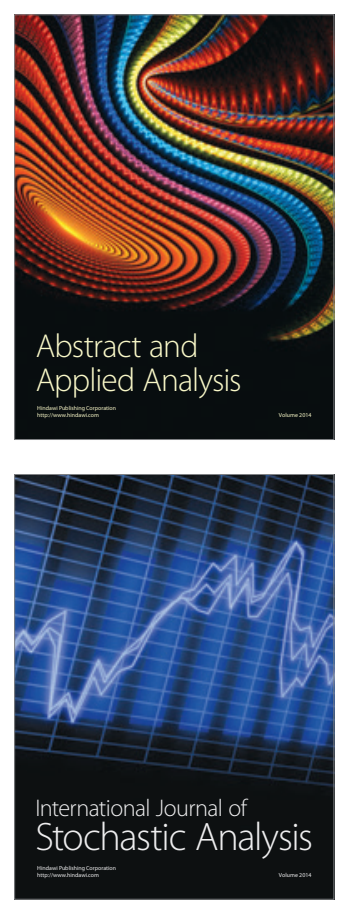

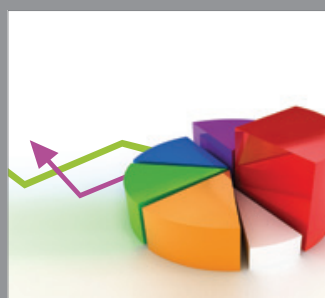

ournal of

Probability and Statistics

Promensencen
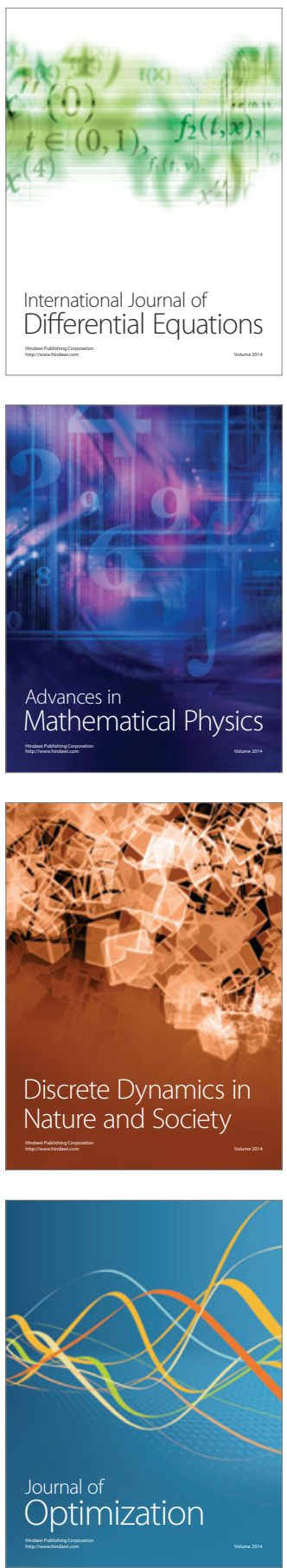